\title{
Synthesising of MgSe Complex Nanoparticle via Bacteria and Characterisation of Fabricated $\mathrm{MgSe} / p$-Si Structure
}

\author{
T. ÇAKICI $^{a, *}$ AND Ö. GÜR ÖZDAL ${ }^{b}$ \\ ${ }^{a}$ Department of Electricity and Energy, \\ Ispir Hamza Polat Vocational School of Higher Education, \\ Ataturk University, Erzurum, Turkey \\ ${ }^{b}$ Department of Biology, Science Faculty, Ataturk University, Erzurum, Turkey
}

Received: 22.12.2020 \& Accepted: 23.04.2021

Doi: 10.12693/APhysPolA.140.9 *e-mail: tuba.cakici@atauni.edu.tr

In this study, we have for the first time reduced complex MgSe nanoparticles to a compound by applying the bacterial synthesizing method using Pseudomonas aeruginosa OG1 strain. Complex MgSe nanoparticles reduced by bacteria in a compound form were grown as thin films on glass and $p$-Si substrates. Electronic, optical, crystal and morphological properties, and chemical composition of these samples were determined by an ultraviolet-visible spectrometer, X-ray D-diffractometer, and field emission-scanning electron microscopy, atomic force microscopy, and electron diffusion X-ray techniques. From the UV-VIS measurements, it was found that the band gap energy of an MgSe thin film sample was $2.53 \mathrm{eV}$. An X-ray D-diffractometer measurement of the MgSe thin film sample grown on the $p$-Si substrate indicated that the thin film sample has a high-quality polycrystalline structure and grains having equal sizes are distributed uniformly. Field emission-scanning electron microscopy and atomic force microscopy measurements demonstrated that the granules forming in the thin film sample are distributed uniformly in the same regions, and also, the granules have regular spherical shapes, sizes, and the same separations and the interface width (rms) values and Ra values, $9.68 \mathrm{~nm}$ and $7.40 \mathrm{~nm}$, are obtained, respectively. The electron diffusion X-ray measurement shows that the weights and atomic weights of materials in this structure are as expected concerning their weights and atomic weights.

topics: nanoparticles, band gap, bacterial synthesis, thin films

\section{Introduction}

Nanomaterial research's multidisciplinary nature has seeded several new and exciting research directions with many possible engineering, medicine, and textiles applications. As new technologies of nanoparticles (NPs) and nanomaterials increase with the developing technology, the synthesis of different and unique particles has gained importance [1]. Nanoparticles acquire new physicochemical properties due to the sizes and quantum properties of nanoparticles [2]. Physical methods, commonly used in nanoparticle production, are costly due to continuous energy consumption to sustain the high pressure and temperature employed in NPs synthesis and need highly advanced devices. Also, the synthesis of many nanoparticles using chemical methods has an advantage, but this method leads to toxic and non-eco-friendly byproducts [3].

The number of commercial products manufactured with nanoparticles is positively increasing, and also their popularity is growing rapidly. With the increasing demand and environmental impact of various nanomaterials obtained with traditional methods containing harmful additives that have resulted in unprecedented physicochemical structures of various NPs, there is a need for nanomaterials obtained with green methods which are not only cost-effective but also eco-friendly to the users and the environment. Furthermore, thin film deposition procedures can profit from minimal heating without the use of any toxic gases.

Green synthesis approaches to the production of metallic nanoparticles and films have been a highly engaging research area in nanomaterials development in recent years. Different types of biological samples such as algae, bacteria, plants, and fungi can be used as possible eco-friendly alternatives instead of the physical and chemical processes for the green synthesising of metallic nanoparticles [4-7]. Microbial synthesis has developed as a promising alternative to traditional nanoparticle synthesis methods. Microbial synthesis provides a cheap (minimum costs and low energy), safe, simple, high-yield and nontoxic way for nanoparticles synthesis. Microbial nanoparticle syntheses are realised by various bio-reducing agents such as enzymes, terpenoids, peptides, proteins, 
organic acids, exopolysaccharides and electron shuttle quinines [5, 8-10]. Besides, the use of bacteria for the growth of NPs has the added advantage with a potential to allow for a controlled growth after the functionalisation of their environment.

Recently, heterostructures made of II-VI compound semiconductors having wide bandgaps have attracted significant attention due to their properties and feasibility in laser diodes [11] and many types of optoelectronic devices [12]. In recent years, many researchers have focused on preparing nanostructured semiconductors with novel electrical and optical properties by using economically inexpensive and straightforward applicable methods $[13,14]$. The alkaline chalcogenides materials group, such as CuSe, CaSe, ZnSe, and MgSe, has significantly impacted their potential applications in various optoelectronic devices. However, those in this group which display luminescence properties have become more and more critical [15]. On the other hand, the group II-VI selenite quantum well structures attract great interest of scientific communities studying semiconductor devices such as quantum cascade structures, short-wavelength quantum well-infrared photodetectors, yellow laser diodes, and yellow emitters [16]. Notably, MgSe quantum well structures formed with the selenide group have been asserted as an attractive alternative as compared to the other semiconductor systems for the optical communication wavelength of $1.55 \mathrm{~mm}[17,18]$. Among various metal selenides, MgSe is one of the essential members of semiconductors belonging to this family with a wide band gap prepared with various sizes and Np geometries.

For the first time in this study, MgSe nanoparticles were produced using the bacterial biosynthesised method based on Pseudomonas aeruginosa OG1 strain, and subsequently analysed. Firstly, MgSe NPs were obtained, and then nanocrystalline MgSe films were formed on glass and $p$-Si substrates sintered for obtaining a high quality of thin films. The optical, structural and morphological chemical composition characterizations of these thin film samples were performed by the UV-VIS spectrometer, X-ray D-diffractometer (XRD), and field emission-scanning electron microscopy (FE-SEM), atomic force microscopy (AFM), and electron diffusion X-ray (EDX) techniques.

\section{Experimental}

\subsection{Obtaining MgSe NP solution} by bacterial synthesised method

We realised this study in two steps. In the first step, we obtained MgSe nanoparticles, using the green method that does not cause harmful additives in the products resulting from chemicals, as compared to the traditional methods. We fabricated an $\mathrm{Ag} / \mathrm{MgSe} / p$-Si $/ \mathrm{Al}$ device using produced MgSe nanoparticles as an interlayer in the second step. For MgSe nanoparticles' production, Pseudomonas aeruginosa OG1 strain was incubated for $24 \mathrm{~h}$ at $150 \mathrm{rpm}$ and $30^{\circ} \mathrm{C}$ in Tryptic Soy Broth. The bacterial suspension (100 $\mu$ l, OD600 1) was inoculated into Luria Bertani Broth medium (20 ml) and then $0.5 \mathrm{mM} \mathrm{MgSO}_{4}$ and $0.5 \mathrm{mM} \mathrm{Na}_{2} \mathrm{O}_{3} \mathrm{Se}$ were added. A bacterial nanoparticle biosynthesis was performed on a rotary shaker $(150 \mathrm{rpm})$ in the dark conditions for $96 \mathrm{~h}$ at $30^{\circ} \mathrm{C}$. This solution was prepared in double-distilled water.

After the incubation period, the solution was purified, separating the MgSe nanoparticles from unwanted contaminants and bacteria cells. Cell suspensions containing nanoparticles were first sonicated for $5 \mathrm{~min}$ at $100 \mathrm{~W}$ in an ultrasonic bath (Elma/S30) and then centrifuged at $10000 \mathrm{rpm}$ for $10 \mathrm{~min}$. This solution was then divided into two different beakers. One of these solutions was used to manufacture an MgSe thin film onto the glass substrate whose UV-VIS measurement will be performed, and the other was used for making an $\mathrm{MgSe} / p$-Si structure onto $p$-Si substrate whose XRD, AFM, FE-SEM and EDS measurements will be performed.

\subsection{Fabrication of MgSe thin films on glass and $p$-Si substrates}

After preparing solutions as above, we formed the MgSe thin films onto glass and $p$-Si substrates for two different purposes. Firstly, we fabricated the MgSe thin film onto the glass substrate to obtain the MgSe thin film sample's band gap energy. To do it, we dropped a few drops on the glass substrate and left it to dry at $65^{\circ} \mathrm{C}$. Hence, the MgSe thin film was formed. The other thin film samples are the samples that are fabricated onto $p$-Si substrates. The $p$-Si wafer was sliced into six pieces with $15 \times 10 \mathrm{~mm}^{2}$. Then they were degreased with acetone and methanol in an ultrasonic cleaner for $10 \mathrm{~min}$ and consecutively they were etched in a sequence of $\mathrm{H}_{2} \mathrm{O}: \mathrm{H}_{2} \mathrm{O}_{2}: \mathrm{HNO}_{3}(6: 1: 1)$ at $60^{\circ} \mathrm{C}$, $20 \%$ H.F. and a solution of $\mathrm{H}_{2} \mathrm{O}: \mathrm{H}_{2} \mathrm{O}_{2}: \mathrm{HCl}(6: 1: 1)$ at $60{ }^{\circ} \mathrm{C}, 20 \%$ H.F. We obtained these samples in the same manner as above. The only difference here was the fact that the solution was dropped onto each of the $p$-Si substrates. Five of these samples were used in the XRD, FE-SEM, EDX, and AFM measurements.

\subsection{Characterisation}

Their characterisations were realised after completing the MgSe thin film samples' fabrication on glass and $p$-Si substrates. Firstly, the MgSe thin film sample's optical properties formed onto the glass substrate were determined by an UV-VIS spectrometer (Perkin-Elmer Lambda 2S UV-Visible spectrometer) measurement. On the other hand, XRD Bruker D2 $\left(K_{\alpha}, \lambda=1.54 \AA\right.$, scanning angle $70^{\circ}$ ) was employed to determine the crystal structure and qualitative analysis of the MgSe thin film formed on the $p$-Si substrate. The investigation 
of the samples' surface morphology was performed by AFM [Hitachi 5100N], and FESEM (Sigma 300 Model Zeiss Gemini) measurements and the determination of the chemical composition of the samples was realised by EDX associated with FE-SEM.

\section{Results and discussion}

\subsection{Optical properties of MgSe thin film sample}

The optical absorption spectrum of the MgSe thin film formed onto the glass substrate versus the wavelength in $\alpha$ range varying from 300 to $1000 \mathrm{~nm}$ is shown in Fig. 1. In order to obtain numerical band gap energy value of the MgSe thin film sample, it is necessary to know the absorption coefficient $\alpha$, in terms of the photon's wavelength. The mathematical expression of the transmission of the light passing through a material is given as follows:

$$
I=I_{0} \mathrm{e}^{-\alpha d}
$$

where $I, I_{0}$, and $d$ are the transmitted light intensity, incident light intensity, and thin film sample thickness, respectively. The numerical values of the absorption coefficient $\alpha$ is obtained inserting the experimental values of $I, I_{0}$, and $d$ into (1). The optical band gap energy value of the thin film sample $E_{\mathrm{g}}$ is found by the equation given below [19]:

$$
\alpha h v=A\left(h v-E_{\mathrm{g}}\right)^{n},
$$

where $h v$ and $A$ are the photon's energy and the constant, respectively. In turn, $n$ is the constant having a numerical value of 1 or 2 , corresponding to direct or indirect electron transitions between the valance and conduction bands, respectively. It is known that MgSe has direct transition, therefore $n$ is equal to $\frac{1}{2}$ for this compound. Substituting $\frac{1}{2}$ instead of $n$ in (2) and taking the square of both sides, we obtain $(\alpha h v)^{2}=h v-E_{\mathrm{g}}$ expression. The result of plotting $(\alpha h v)^{2}$ vs $h v$ is seen in Fig. 1, where, in the inset, a curve is obtained. The band gap energy value of the MgSe thin film was found to be $2.53 \mathrm{eV}$, i.e., when applying the extrapolation method to the linear part of the curve (see Fig. 1). This value is equal to the value corresponding to the intercept point of the straight line drawn from the straight part of the curve and with the energy axis $(h v)$. This result is in good agreement with [20].

\subsection{Structural and morphological properties of MgSe thin film formed on $p$-Si substrate}

Figure 2 shows the XRD patterns of the MgSe thin film deposited onto the $p$-Si substrate. Two peaks appear at (111) and (200) along the directional planes. According to the data obtained from (JCPDS card No. 18-0777), these crystal phases show that the MgSe thin film has a cubic lattice. These observed patterns are in good agreement with similar results obtained from different studies in the literature [21-23]. The most substantial peak belongs to $p$-Si appearing at (100) directional plane

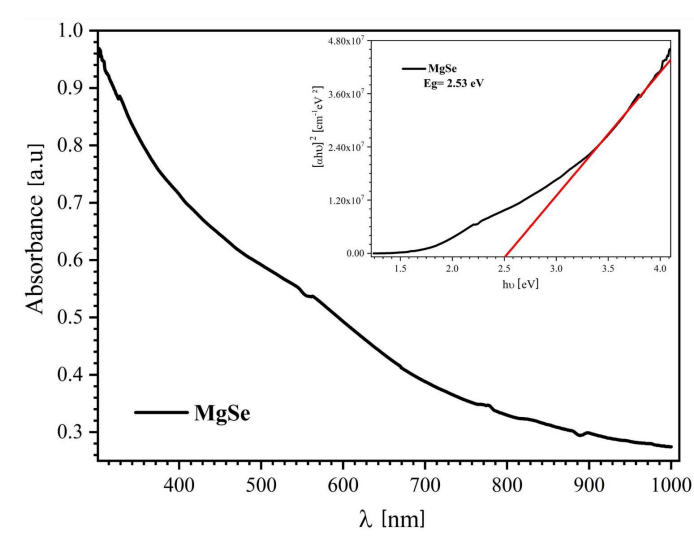

Fig. 1. The plot of optical absorbance versus wavelength, $\lambda[\mathrm{nm}]$ and plot of $(\alpha h v)^{2}$ versus $h v$ (inset) for as-deposited MgSe film onto glass substrate at room temperature.

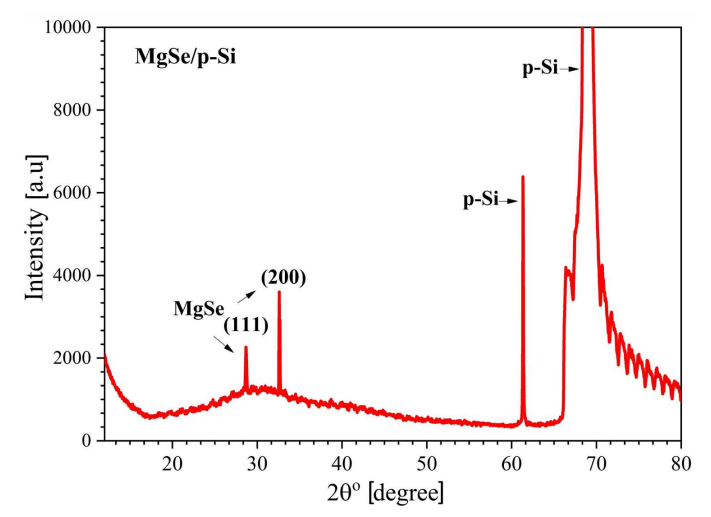

Fig. 2. XRD patterns of MgSe thin film deposited on the $p$-Si substrate.

with cubic crystallization. Structural parameters of the MgSe thin film grown on the $p$-Si substrate, such as average crystallite size $D$, and the distance between the planes $d$, were calculated using the recorded XRD patterns and with the help of the well-known Scherrer equation [21-24] given as

$$
D=\frac{0.9 \lambda}{\beta \cos (\theta)} \text {. }
$$

The calculated values of $D$ and $d$ structural parameters are given in Table I.

Surface morphology of the MgSe $/ p$-Si structure was investigated using the data obtained from the AFM and SEM measurements. AFM is an effective method used for analysing the surface morphology of thin films. Figure $3 \mathrm{a}$ and $\mathrm{b}$ shows the $2 \mathrm{D}$ and $3 \mathrm{D}$ AFM images of $\mathrm{MgSe} / p$-Si structure with scales of $2 \mu \times 2 \mu$, respectively. Figure 3 a shows the twodimensional (2D) image of the surface profile of the MgSe thin film. The same sizes of granules appeared in this image, and they are distributed in the same regions uniformly. Importantly, granules have regular spherical shapes, sizes, and separations. 
The structural parameters of the MgSe thin film evaluated on the $p$-Si substrate.

TABLE I

\begin{tabular}{c|c|c|c|c|c|c|c}
\hline \hline$(h k l)$ & FWHM & $\begin{array}{c}\text { FWHM } \\
{[\mathrm{rad}]}\end{array}$ & $\begin{array}{c}\text { Intensity } \\
{[\mathrm{a} . \mathrm{u} .]}\end{array}$ & $\begin{array}{c}\text { Observed, } \\
2 \theta\left[^{\circ}\right]\end{array}$ & $\begin{array}{c}d \text {-values } \\
{[\mathrm{nm}]}\end{array}$ & $\begin{array}{c}\text { Crystal size } \\
D[\mathrm{~nm}]\end{array}$ & $\begin{array}{c}\text { Crystal } \\
\text { phase }\end{array}$ \\
\hline$(111)$ & 0.1122 & 0.00196 & 1102.99 & 28.67 & 3.1102 & 73.03 & cubic \\
$(200)$ & 0.1496 & 0.00261 & 1642.88 & 32.59 & 2.7432 & 55.36 & cubic
\end{tabular}

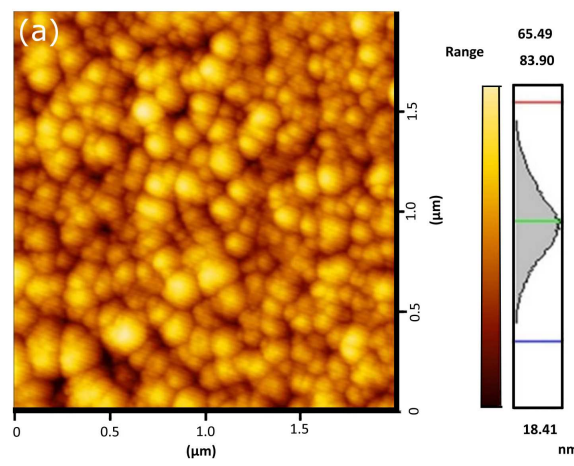

(b)

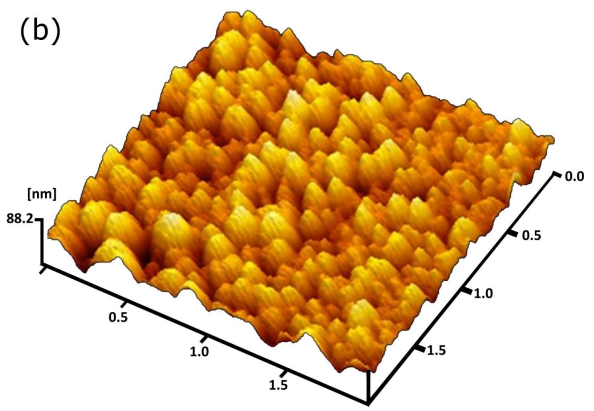

(um)

Fig. 3. AFM images of $\mathrm{MgSe} / p-\mathrm{Si}$ structure (a) $2 \mathrm{D}$ and (b) $3 \mathrm{D}$ image with scale $2 \mu \times 2 \mu$.

In Fig. 3b, grains resembling a cylinder interconnected type are seen. The systems with such a type of morphology are employed in many optoelectronic devices. On the other hand, the height roughness $R a$, and the standard deviation in the surface height within the selected area root mean square (rms) are the most essential two criteria for determining a thin film surface morphology [25]. From Fig. 3b, the interface width (rms) values and $R a$ values are obtained as $9.68 \mathrm{~nm}$ and $7.40 \mathrm{~nm}$, respectively.

Figure 4 illustrates the FE-SEM image of the $\mathrm{MgSe} / p$-Si sample. The MgSe thin film has a uniform and homogeneous surface morphology (see Fig. 4a). The thin film consists of a single type and small densely packed nanocrystals. The grains seen in the image are grains looking like each other, with spherical shapes and distributed uniformly. The average diameter of spherical grains is found to be about $20-50 \mathrm{~nm}$.

This outcome confirms that AFM and SEM results are well-matched with each other. It is also said that the MgSe thin film deposited on the $p$-Si substrate has a high-quality nanocrystal structure. Figure $4 \mathrm{~b}$ shows the EDX chemical composition of
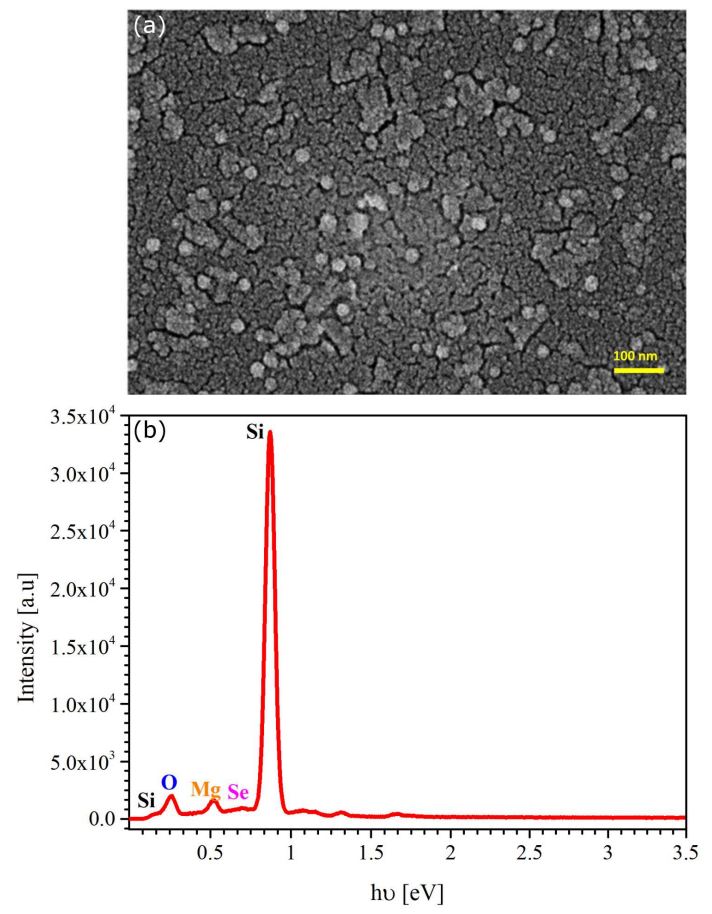

Fig. 4. (a) FE-SEM image and (b) EDX spectrum of $\mathrm{MgSe} / p$-Si structure.

TABLE II

EDX chemical composition of $\mathrm{MgSe} / p$-Si.

\begin{tabular}{c|c|c}
\hline \hline Element & Weight $\%$ & Atomic $\%$ \\
\hline O $K$ & 9.06 & 14.96 \\
$\operatorname{Mg} K$ & 1.32 & 1.44 \\
Se $L$ & 1.15 & 0.39 \\
Si $K$ & 88.47 & 83.22
\end{tabular}

the MgSe thin film formed onto the $p$-Si substrate. From the EDX measurement, it can be said that the bacteria produced MgSe nanoparticles.

In Fig. 4b, the EDS measurement graphic is presented. One can see that EDX measurements show that the weights and atomic weights of materials in this structure are as expected (see Table II).

\section{Conclusion}

MgSe nanoparticles were synthesised via the bacterial green biosynthesis method based on Pseudomonas aeruginosa OG1 strain for the first time by us. Then, nanocrystalline MgSe thin film samples were deposited on $p$-Si and glass substrates. In the latter, interfacial thin films in $\mathrm{Ag} / \mathrm{MgSe} / p-\mathrm{Si} / \mathrm{Al}$ 
device architectures were fabricated. Then, firstly, the thin film sample's optical property grown on glass was determined by UV-Vis. The other characteristic parameters of the $\mathrm{MgSe} / p$-Si sample, such as crystal structure, surface morphology, and chemical composition, were investigated by XRD and FE-SEM AFM, with EDS techniques. These results proved that the MgSe thin film sample has a polycrystalline structure in nanoscale grain size distributed uniformly.

These results showed that MgSe nanoparticles could be produced using the biosynthesising assisted method, a low-cost alternative to other chemical and physical methods. Besides, in this study, we grew the MgSe thin film on the $p$-Si substrate. This structure is a heterostructure and because of that it can be turned into an $\mathrm{Ag} / \mathrm{MgSe} / p-\mathrm{Si} / \mathrm{Al}$ structure making some modifications. As a result, this structure may be employed in different industrial applications such as photodiodes, photosensors, and quantum well laser diodes. Therefore, we plan to fabricate this structure in our future study and investigate the $I-V$ and $C-V$ characteristics in detail.

\section{References}

[1] G. Chen, I. Roy, C. Yang, P.N. Prasad, Chem. Rev. 116, 2826 (2016.

[2] M.R. Bindhu, M. Umadevi, M.K. Micheal, M.V. Arasu, N.A. Al-Dhabi, Mater. Lett. 166, 19 (2016).

[3] A.K. Mittal, J. Bhaumik, S. Kumar, U.C. Banerjee, J. Coll. Interface Sci. 415, 39 (2014).

[4] J. Singh, T. Dutta, K.H. Kim, M. Rawat, P. Samddar, P. Kumar, J. Nanobiotechnol. 16, 84 (2018).

[5] P. Singh, Y.J. Kim, D. Zhang, D.C. Yang, Trends Biotechnol. 34, 588 (2016).

[6] G. Gahlawat, A.R. Choudhury, $R S C A d v$. 9, 12944 (2019).

[7] M. Ozdal, O.G. Ozdal, O.F. Algur, Pol. J. Microbiol. 65, 63 (2016).

[8] T. Çakıcı, M. Ozdal, M. Kundakcı, R. Kayall, Mater. Sci. Semicond. Process. 103, (2019) 104610.
[9] M. Kitching, M. Ramani, E. Marsili, Microb. Biotechnol. 8, 904 (2015).

[10] S.A. Kumar, M.K. Abyaneh, S.W. Gosavi, S.K. Kulkarni, R. Pasricha, A. Ahmad, M.I. Khan, Biotechnol. Lett. 29, 439 (2007).

[11] H. Morkoc, S. Strite, G.B. Gao, M.E. Lin, B. Sverdlow, M. Burns, J. Appl. Phys. 76, 1363 (1994).

[12] R.F.C. Farrow, G.R. Jones, G.H. Williams, I.M. Young, Appl. Phys. Lett. 39, 954 (1981).

[13] A.U. Ubale, A.N Bargal, Mater. Res. Bull. 46, 1000 (2011).

[14] A.U. Ubale, Mater. Chem. Phys. 121, 555 (2010).

[15] M.W. Wang, M.C. Phillips, J.F. Swenberg, E.T. Yu, J.O. McCaldin, T.C. McGill, J. Appl. Phys. 73, 4660 (1993).

[16] A. Shen, G. Chen, K. Zhao, J.T. Lai, M.C. Tamargo, J. Vac. Sci. Technol. B 31, $03 \mathrm{C} 103$ (2013).

[17] G. Chen, M.C. Tamargo, A. Shen, J. Appl. Phys. 118, 165707 (2015).

[18] J. De Jesus, G. Chen, L.C. HernandezMainet, A. Shen, M.C. Tamargo, J. Cryst. Growth 425, 207 (2015).

[19] J. Tauc, The Optical Properties of Solids, Academic Press, New York 1966, p. 277.

[20] A.U. Ubale, Y.S. Sakhare, S.G. Ibrahim, M.R. Belkhedkar, Solid State Sci. 23, 96 (2013).

[21] A.U. Ubale, Y.S. Sakhare, Mater. Sci. Semicond. Process. 16, 1769 (2013).

[22] A.U. Ubale, Y.S. Sakhare, Vacuum 99, 124 (2014).

[23] Y.S. Sakhare, N.R. Thakare, A.U. Ubale, J. Phys. Math. 2, 17 (2016).

[24] T. Çakıcı, B. Güzeldir, M. Sağlam, J. Alloys Compd. 646, 954 (2015).

[25] D. Raoufi, A. Kiasatpour, H.R. Fallah, A.S.H. Rozatian, Appl. Surf. Sci. 253, 9085 (2007). 